\title{
Seasonal variations in Schistosoma haematobium egg excretion in school-age girls in rural KwaZulu-Natal Province, South Africa
}

\author{
E E Christensen, ${ }^{1,2} \mathrm{MD}$; M Taylor, ${ }^{3} \mathrm{PhD} ; \mathbf{S}$ G Zulu, ${ }^{3} \mathrm{MSc}$; K Lillebo, ${ }^{1,2} \mathrm{MD}$; S G Gundersen, ${ }^{4,5} \mathrm{MD}, \mathrm{PhD} ; \mathbf{S}$ Holmen, ${ }^{1,2} \mathrm{MD}, \mathrm{PhD}$ \\ E Kleppa, ${ }^{1,2} \mathrm{MD}$, PhD; B J Vennervald, ${ }^{6} \mathrm{MD}$, MSA, PhD; P D Ndhlovu, ${ }^{7} \mathrm{MSc}, \mathrm{PhD}$; E F Kjetland, ${ }^{1,3} \mathrm{MD}, \mathrm{PhD}$ \\ ${ }^{1}$ Norwegian Centre for Imported and Tropical Diseases, Department of Infectious Diseases Ullevaal, Oslo University Hospital, \\ Norway \\ ${ }^{2}$ Faculty of Medicine, University of Oslo, Norway \\ ${ }^{3}$ Discipline of Public Health Medicine, Nelson R Mandela School of Medicine, School of Public Health, College of Health Sciences, \\ University of KwaZulu-Natal, Durban, South Africa \\ ${ }^{4}$ Department of Global Development and Planning, Faculty of Social Sciences, University of Agder, Kristiansand, Norway \\ ${ }^{5}$ Research Department, Sørlandet Hospital HF, Kristiansand, Norway \\ ${ }^{6}$ Section for Parasitology and Aquatic Diseases, Faculty of Health and Medical Sciences, University of Copenhagen, Denmark \\ ${ }^{7}$ Imperial College London, UK
}

Corresponding author: E E Christensen (erikegelandchristensen@gmail.com)

\begin{abstract}
Background. A predominant feature of Schistosoma haematobium infection is urinary egg excretion, and microscopic egg detection remains the accepted standard field diagnostic tool. Praziquantel is the drug of choice for schistosomiasis, and the World Health Organization recommends that it should be administered to all children $>4$ years of age living in schistosomiasis-endemic areas. The frequency of mass drug administration depends on the prevalence rate in the community. Urinary schistosome egg output has a day-to-day and hour-to-hour intrasubject variation. Therefore, it is important to assess possible seasonal variations in egg excretion to improve the planning of drug treatment.

Objectives. To assess the influence of seasonality on urinary schistosome egg excretion in South Africa (SA).

Methods. We performed a prospective cohort study, exploring seasonal variations of S. haematobium egg excretion in 184 girls aged 10 - 12 years from randomly selected schools in a rural area of KwaZulu-Natal Province, SA. The area has a subtropical climate characterised by a cool dry season and a hot humid season. For children, water contact is higher in the latter season. At baseline, 108 girls were examined in the hot season, and 76 in the cold season. In the next year's cold season the untreated patients were re-investigated before treatment.

Results. There was a decrease in infection in the group initially tested in the hot season compared with the group tested in the cold season at both time points when adjusted for age and water contact (adjusted odds ratio 3.61 (95\% confidence interval 1.14 - 11.44); $p=0.03$ ).

Conclusions. This unique study shows that schistosomiasis prevalence determined by microscopy exhibits seasonal variation, with a higher prevalence in the hot rainy season. Precise community prevalence estimations are key in decisions to treat communities. There was significantly lower egg output in the cold season, and sampling in that season may therefore underestimate the prevalence of urinary schistosomiasis. The study indicates that sampling in SA should be done in the hot season.
\end{abstract}

S Afr Med J 2018;108(4):352-355. DOI:10.7196/SAMJ.2018.v108i4.12775

Worldwide an estimated 218 million people have schistosomiasis, of whom $\sim 90 \%$ live in Africa. ${ }^{[1]}$ Schistosomiasis is a parasitic disease caused by Schistosoma trematodes. ${ }^{[1]}$ It is endemic in KwaZulu-Natal (KZN) Province, South Africa (SA), and Schistosoma haematobium is the most common species. ${ }^{[2]}$ S. haematobium causes urogenital schistosomiasis, with symptoms including stunting, infertility, cancer, hydronephrosis and increased susceptibility to HIV. ${ }^{[1,3]}$ Other schistosome species, the most common being S. mansoni and S. japonicum, ${ }^{[3]}$ are not endemic in $\mathrm{KZN}^{[2]}$ Mass drug administration with praziquantel is the primary tool for combating schistosomiasis, and school-age children are the main target group. The frequency of mass drug administration depends on a community's precontrol prevalence rate, which is assessed by parasitological methods or questionnaires, differentiating into low $(<10 \%)$, moderate $(10-50 \%)$ and high risk $(>50 \%)$ communities. ${ }^{[4]}$ Egg counts by microscopic examination of urine remain the accepted standard diagnostic tool for detecting $S$. haematobium infection. ${ }^{[5,6]}$ When egg excretion is low, the sensitivity of urine microscopy for ova is poor. Consequently, communities' need for intervention might be underestimated. ${ }^{[3]}$

There are seasonal variations in the transmission of schistosomiasis in infested water sources, ${ }^{[7]}$ also in SA. ${ }^{[2,8]}$ Furthermore, there are intrasubject variations in S. haematobium egg output. ${ }^{[9-11]}$ A few, albeit small, studies indicate that egg excretion may vary, depending on the season, but none of these has been done in a country with a subtropical climate, such as SA. ${ }^{[12-14]}$ As community prevalence is the basis for decisions to treat, it is important to identify the optimal period for assessment of egg excretion and community prevalence. In anticipation of planned control programmes in SA, the objective of this observational study was to assess the influence of season on urinary schistosome egg excretion.

\section{Methods}

Study population, area and climate

The study was conducted in the rural inland Ugu District, SA. In 2011, $16.1 \%$ of the district's total population did not have access to piped water, and people relied on unsafe water from nearby rivers. ${ }^{[15]}$ The area has a subtropical climate, characterised by hot and humid summers (December through April), and dry and cool winters (June through August) (Fig. 1) ${ }^{[16,17]}$ For children, water contact is higher in summer, especially from mid-December to mid-January, which is a school holiday period in this region. ${ }^{[17]}$ 


\section{Study inclusion and sampling}

In 2010, baseline data were collected from girls aged 10 - 12 years, who were attending 18 randomly selected schools. The schools were visited in a random fashion. All girls 10 - 12 years of age were invited to take part in the study, and 1057 gave consent (Fig. 2). This cohort was initially selected for studies on female genital schistosomiasis and hence no boys were recruited. From February to April (hot season) and June to November (cold season), 970 of these girls submitted a urine sample (omitting December, January and May owing to school holidays, examinations or examination-related activities). ${ }^{[18]}$ A total of 940 participants had reliable age data (Fig. 2). Only one case of S. mansoni was found at baseline. ${ }^{[18]}$

In 2011, all 18 schools were revisited in the cold season (from end of May to September). Nurses from the Department of Health offered a single dose of praziquantel $40 \mathrm{mg}$ per $\mathrm{kg}$ of body weight. ${ }^{[15]}$ For research purposes, every third pupil was asked for a urine sample on the same day. One school did not provide urine samples. Some participants could not be found and some did not provide urine samples (Fig. 2). In total, 184 of the participants submitted urine samples at follow-up, which were collected in the cold season.

Of the 184 participants who submitted a urine sample at two time points, baseline urine specimens from a subgroup were collected in the hot season (February - April; group 1, $n=108$ ) or cold season (June November; group 2, $n=76$ ) for follow-up later in the cold season (Fig. 2).

At baseline, the participants were interviewed face-to-face in isiZulu on water contact, schistosomiasis infection (local name: isichenene) and if they had received prior treatment. All urine specimens were collected between $10 \mathrm{~h} 00$ and $14 \mathrm{~h} 00$ and all were processed by the same method as described previously. ${ }^{[18]}$ The participants were not given fluids before sampling.

\section{Data management and statistical analyses}

Data were entered into Excel (Microsoft Corp., USA) and subsequently imported into SPSS Version 22 (IBM Corp., USA),

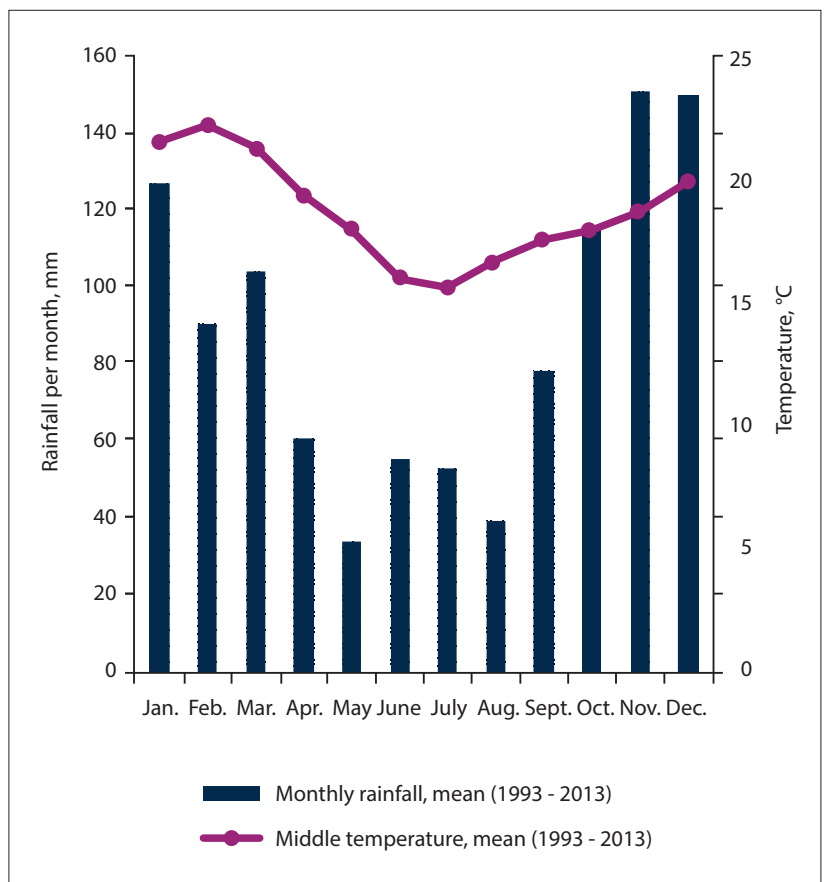

Fig 1. Meteorological registrations, Paddock weather station (nearest weather station). ${ }^{[16]}$

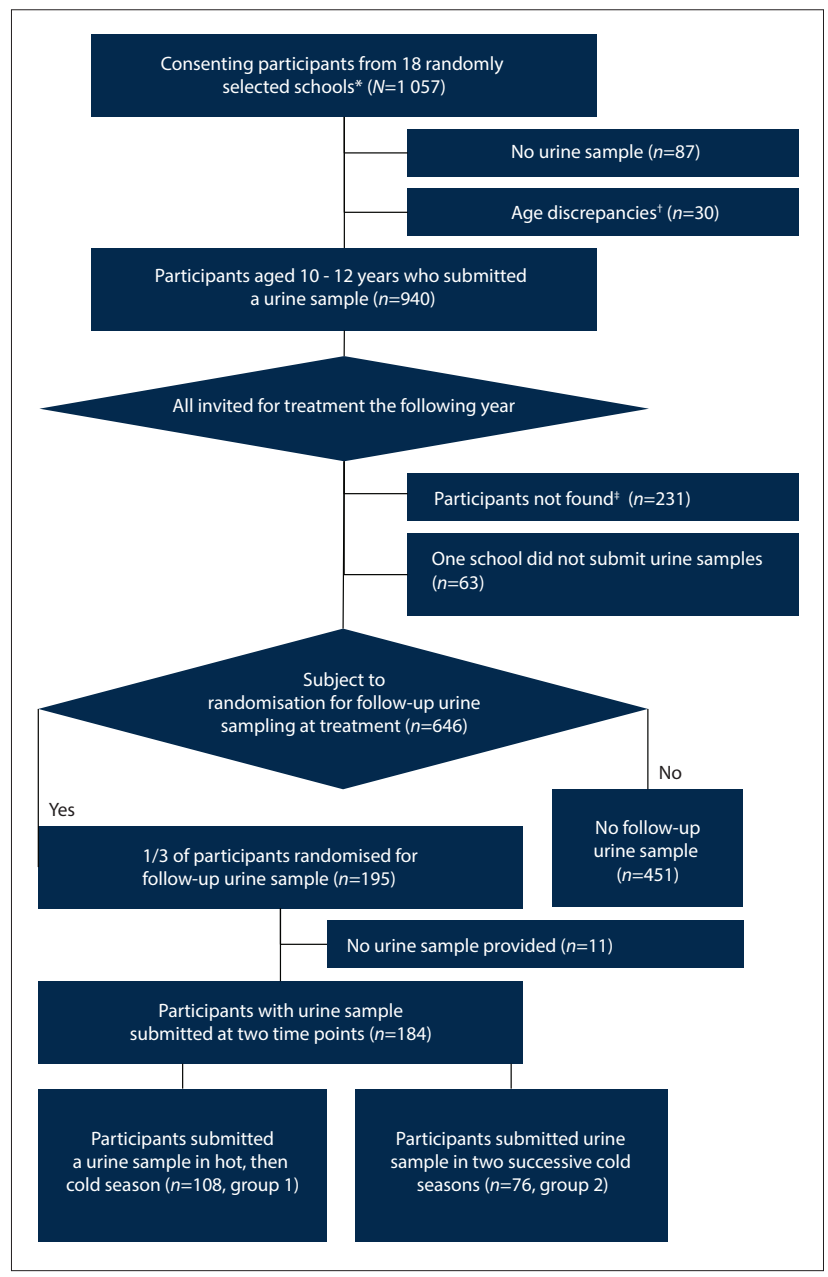

Fig. 2. Flowchart showing selection and randomisation of participants. $\left({ }^{*}\right.$ Only girls, as this was part of a study on female genital schistosomiasis. ${ }^{\dagger}$ Provided irreconcilable dates of birth and were found to be up to 4 years older by the new dates provided. These were excluded as urinary schistosomiasis egg excretion is age-dependent. ${ }^{[19] *}$ Teachers/peers were not asked about reasons for the participants' absence.)

using two-pass verification for quality control. Odds ratios (ORs) with 95\% confidence intervals (CIs) were used to compare schistosomiasis prevalence, previous water contact or treatment in groups 1 and 2 . The Mann-Whitney $U$-test was used to compare the duration between baseline and follow-up, because of the non-normally distributed continuous data. For normally distributed continuous data (age), Student's $t$-test was used. For paired data (baseline and follow-up), the Wilcoxon signed-rank test was used for non-normally distributed continuous data (urine S. haematobium intensity) and McNemar's test for nominal data (prevalence). To study the impact of several variables simultaneously, logistic regression analysis was applied at a $5 \%$ significance level; variables were included if the $p$-value from crude association was $<0.2$ and if the Spearman rank correlation coefficient was $<0.7$.

\section{Ethical approval}

The project was approved by the Biomedical Research Ethics Committee, University of KwaZulu-Natal (ref. no. BF029/07), the Department of Health, Pietermaritzburg (ref. no. HRKM010-08), the Norwegian Regional Committee for Medical and Health Research Ethics (ref. no. 469-07066a1.2007.535) and the European Group on 
Ethics in Science and New Technologies (ref. no. IRSES-2010:269245). The departments of Health and Education in Ugu District granted permission. ${ }^{[15,18]}$ The participants were included only if a guardian gave written consent and the participant assented. Antihelminthic treatment was delayed owing to lack of access to praziquantel. ${ }^{[20]}$ As soon as praziquantel could be accessed, treatment was offered free by the Department of Health. The medical consequences of the delay are unknown.

\section{Results}

\section{General characteristics}

A total of 940 girls aged $10-12$ years submitted urine samples. A median of 42 (range 7 - 171) girls were included from each of the 18 schools. The mean age was 11.1 (standard deviation (SD) 0.8 ) years. At baseline, $27.6 \%$ (259/940) were S. haematobiumpositive, and in the positive group the geometric mean count was 23 eggs $/ 10 \mathrm{~mL}$ urine.

\section{Follow-up study}

Of the girls who were examined at baseline, 184 were re-examined during the following year's cold season. The median interval between the examinations was 14.8 (7.3 - 15.6) months. There had been no mass treatment in the interim.

Urine specimens from 108 participants were initially submitted in the hot season of 2010, with second urine specimens in the cold season of the following year (Table 1, group 1). Moreover, urine samples from 76 participants collected in the cold season of 2010 (group 2) were compared with a second urine specimen collected during the next cold season. Thus, group 2 submitted urine specimens in two cold seasons. There was no significant difference in age between the two groups (Student's $t$-test, $p=0.662$ ). The interval period was somewhat longer for the participants tested in the hot season at baseline (group 1, median 15.3 (14.3 - 15.7) months) than for those tested in the cold season at baseline (group 2, median 12.0 (7.3 - 12.1) months; Mann-Whitney $U$-test, $p<0.001)$.

At follow-up, $22.3 \%(41 / 184)$ were $S$. haematobium-positive and the geometric mean count was 14 eggs/10 mL urine. In group 1 (participants tested initially in the hot season, then in the cold season), the intensity of urinary schistosomiasis decreased significantly (Wilcoxon signed-rank test, $p=0.05$ ). In group 1 , the prevalence at baseline was $38 \%(41 / 108)$ and at follow-up $29 \%$ (32/108) (McNemar test, $p=0.09$ ). The baseline and follow-up prevalences of group 2 (tested in the cold season on both occasions) did not differ significantly (McNemar test, $p=0.76$ ) - likewise for urinary schistosomiasis intensities (Wilcoxon signed-rank test, $p=0.39$ ) (Table 1).

Interestingly, as shown in Fig. 3, there was no significant difference in conversion to positive schistosomiasis status in the groups after adjusting for age and water contact (adjusted OR 1.77 (95\% CI 0.47 - 6.67); $p=0.40$ ). Conversion to negative schistosomiasis status was more common in those who were examined in the hot season initially than in those who were tested in the cold seasons both times (adjusted OR 3.61 (95\% CI 1.14 - 11.44); $p=0.03$ ). The girls who were tested in the hot season initially had a higher schistosomiasis prevalence, more water contact, and more self-reported schistosomiasis (Table 1).

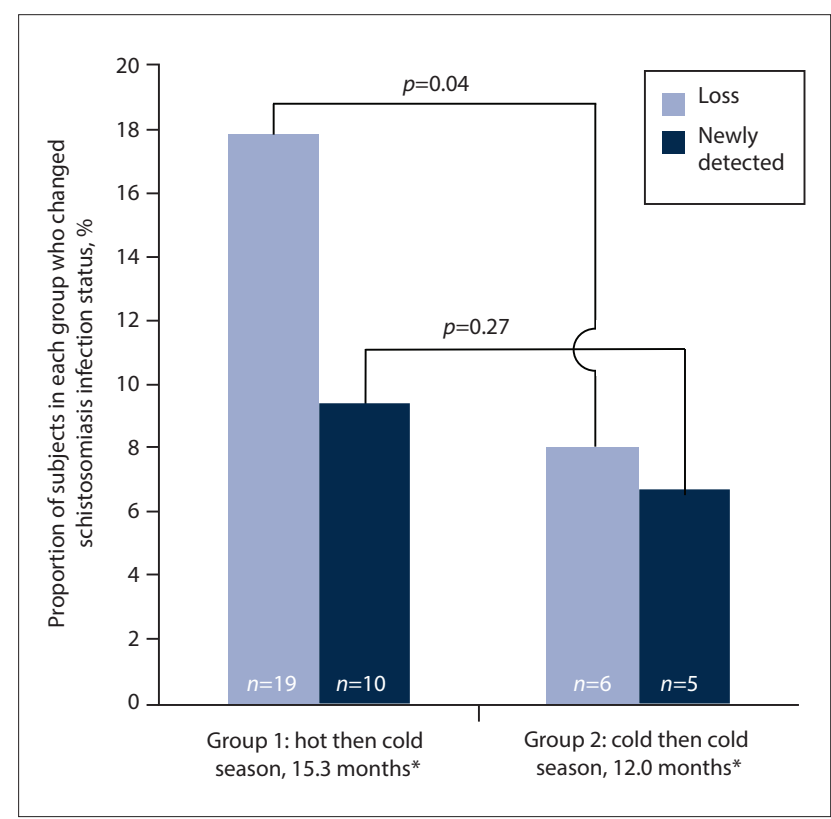

Fig. 3. Proportion of subjects who changed Schistosoma haematobium infection status between baseline and follow-up. $\left({ }^{*}\right.$ Median duration between baseline and follow-up.)

\begin{tabular}{|c|c|c|c|c|}
\hline & $\begin{array}{l}\text { Baseline urine } \\
\text { collected in hot season } \\
\text { (group 1) }\end{array}$ & $\begin{array}{l}\text { Baseline urine } \\
\text { collected in cold season } \\
\text { (group 2) }\end{array}$ & OR $(95 \% \mathrm{CI})$ & $p$-value \\
\hline Participants, $n(\%)$ & $108(58.7)$ & $76(41.3)$ & - & - \\
\hline Schools, $n$ & 10 & 7 & - & - \\
\hline Reported having had schistosomiasis, $n$ (\%) & $36 / 105(33.3)$ & 9/73 (11.8) & $3.7(1.7-8.3)$ & 0.001 \\
\hline Reported prior treatment for schistosomiasis, $n$ (\%) & 20/108 (18.5) & $4 / 72(5.3)$ & $4.1(1.3-12.5)$ & 0.014 \\
\hline Reported freshwater contact, ${ }^{* \dagger} n(\%)$ & $90 / 108(83.3)$ & $26 / 76(34.2)$ & $9.6(4.8-19.2)$ & $<0.001$ \\
\hline \multicolumn{5}{|l|}{ Prevalence, $\%$} \\
\hline Baseline & 38.0 & 13.2 & $4.0(1.9-8.7)$ & $<0.001$ \\
\hline Follow-up ${ }^{*}$ & 29.6 & 11.8 & $3.1(1.1-7.0)$ & 0.006 \\
\hline \multicolumn{5}{|l|}{ Intensity $(\text { eggs } / 10 \mathrm{~mL})^{\varsigma}$} \\
\hline Baseline & 18.4 & 9.0 & $1.6(1.2-2.1)$ & 0.001 \\
\hline Follow-up & 14.9 & 12.9 & $1.4(1.1-1.9)$ & 0.012 \\
\hline
\end{tabular}




\section{Discussion}

In this novel study of untreated children over a period of $>1$ year in rural SA, we found that the S. haematobium prevalences and intensities were lower in the cold season than in the hot season. This may indicate that urine sampling in the hot season in this subtropical climate yields higher prevalences and intensities, which could have an impact on the decision to provide mass treatment.

Seasonal water contact patterns and snail density fluctuations may affect the transmission rate of $S$. haematobium, but it is not known if ovum excretion has a parallel presentation. ${ }^{[7,12,14]}$ The worm lives for a mean period of 5 years; there are also numerous reports of live ova excretion many years after exposure, indicating worm longevity and persistent ability to lay eggs. ${ }^{[3]}$ Low prevalence and intensity in the cold season are more likely to be caused by a lower ability to lay eggs rather than a number of worms dying during the first year of infection. Evolution of antifecundity immunity during the cold season is a possible source for reduction in the observed egg excretion. ${ }^{[21]}$ If this is the case, the detection rates might be highest in the weeks after worm maturation, $\sim 6$ weeks after infection. The low ova excretion and thus lower microscopy sensitivity may lead to a lower observed prevalence. The subtropical climate of SA differs from most areas usually endemic of schistosomiasis, as it has a longer cold season with less water contact. We would argue that a long cold season would probably decrease the observed prevalence more than a shorter season with low water contact.

While polymerase chain reaction-based assays and circulating anodic antigen (CAA) tests are both more sensitive alternatives to urine microscopy, they are either too costly in a field context or not available commercially. ${ }^{[6,22]}$ Therefore, urine microscopy for $S$. haematobium ova continues to be a preferred field diagnostic test. However, our results indicate that urine microscopy should be done during the hot season in SA, ensuring as little underestimation of prevalence as possible.

Repeated investigations without treatment are usually not done, as it is unethical to leave people untreated. However, a few other, albeit small, studies indicate that egg excretion may vary through the seasons of a year. ${ }^{[12,13,23,24]}$ We can possibly preclude a decrease in agedependent schistosomiasis prevalence, as the age of peak prevalence is expected later in moderate endemic areas. ${ }^{[19,25]}$ We cannot preclude that some participants might have sought treatment between the two examinations, although only $15 \%$ reported having received such treatment before baseline in local clinics. ${ }^{[18]}$ We also cannot preclude that the participants were dehydrated in the hot season and therefore yielded higher egg outputs, although water was not offered at any sampling. Furthermore, the two groups are different, even though they were visited in a random sequence.

\section{Conclusions}

This study indicates that microscopy of urine collected in the cold season might cause an underestimation of the prevalence in SA. This would lead to an underestimation of the need for treatment. In the current absence of more sensitive field diagnostics for $S$. haematobium, the use of urine microscopy remains the standard field diagnostic test. To ensure correct prevalence rates and correct interventions in schools, we cautiously suggest that the best time for collection of urine samples is the hot season. Further research is needed to explore if the situation is similar in boys and in different climatic areas.

Acknowledgements. The authors would like to thank the children who participated in the study, their parents, guardians and teachers. We are indebted to the Ugu departments of Health and Education, teachers and headmasters of Ugu schools, school health nurses and the research team at the Bilharzia Research Improving Global Health Today (BRIGHT) clinic. Author contributions. EEC, EFK, SGG and MT designed the study. EEC, SH and EFK analysed the data. EEC, SGZ, EK, EFK and KL collected the data. EEC wrote the original manuscript and managed the database. MT, SGZ, KL, SGG, SH, EK, BJV, PDN and EFK reviewed and edited the manuscript.

Funding. This study was funded by a research grant from the European Council, Norwegian Centre for Imported and Tropical Diseases and the Bill and Melinda Gates Foundation.

Conflicts of interest. None.

1. World Health Organization. Schistosomiasis, Fact Sheet No. 115. Geneva: WHO, 2017. http://www. who.int/mediacentre/factsheets/fs115/en/ (accessed 1 October 2017).

2. Saathoff E, Olsen A, Magnussen P, et al. Patterns of Schistosoma haematobium infection, impact of praziquantel treatment and re-infection after treatment in a cohort of schoolchildren from rural KwaZulu-Natal/South Africa. BMC Infect Dis 2004;4:40. https://doi.org/10.1186\%2F1471-2334-4-40 3. King C, Dangerfield-Cha M. The unacknowledged impact of chronic schistosomiasis. Chronic Illn 2008;4(1):65-79. https://doi.org/10.1177\%2F1742395307084407

4. World Health Organization. Preventative Chemotherapy in Human Helminthiasis. Coordinated use of Antihelminthic Drugs in Control Interventions: A Manual for Health Professionals and Programme Managers. Geneva: WHO, 2006.

5. Mutapi F. Improving diagnosis of urogenital schistosome infection. Expert Rev Anti Infect Ther 2011;9(10):863-865. https://doi.org/10.1586\%2Feri.11.101

6. Rollinson D, Knopp S, Levitz S, et al. Time to set the agenda for schistosomiasis elimination. Acta Trop 2013;128(2):423-440. https://doi.org/10.1016\%2Fj.actatropica.2012.04.013

Augusto G, Magnussen P, Kristensen T, Appleton C, Vennervald B. The influence of transmission season on parasitological cure rates and intensity of infection after praziquantel treatment of scason on parasitological cure rates and intensity of infection after praziquantel treatment of Schistosoma haematobium-infected schoolchildren in Mozambique. Parasitology 2009;136(13):1771-
1779. https://doi.org/10.1017/s0031182009006210 1779. https://doi.org/10.1017/s00311820090062

. Pitchford RI Viser PS. Some further observations on schistosome transmission in the Eastern Transvaal. Bull World Health Organ 1965;32(1):83-104

9. Braun Munzinger RA, Southgate BA. Repeatability and reproducibility of egg counts of Schistosoma haematobium in urine. Trop Med Parasitol 1992;43(3):149-154

10. Warren KS, Siongok TK, Houser HB, Ouma JH, Peters PA. Quantification of infection with Schistosoma haematobium in relation to epidemiology and selective population chemotherapy. I. Minimal number of daily egg counts in urine necessary to establish intensity of infection. J Infect Dis 1978;138(6):849855. https://doi.org/10.1093/infdis/138.6.849

11. Savioli L, Hatz C, Dixon H, Kisumku UM, Mott KE. Control of morbidity due to Schistosoma haematobium on Pemba Island: Egg excretion and hematuria as indicators of infection. Am J Trop Med Hyg 1990;43(3):289-295. https://doi.org/10.1016\%2F0035-9203\%2889\%2990336-2

12. Wilkins HA, Scott A. Variation and stability in Schistosoma haematobium egg counts: A four-year study of Gambian children. Trans R Soc Trop Med Hyg 1978;72(4):397-404. https://doi.org/10.1016\% 2F0035-9203\%2878\%2990135-9

13. Ciddio M, Mari L, Gatto M, Rinaldo A, Casagrandi R. The temporal patterns of disease severity and prevalence in schistosomiasis. Chaos 2015;25(3):036405. https://doi.org/10.1063\%2F1.4908202

14. Lrevalence in schistosomiasis. Chaos $2015 ; 25(3): 036405$. https://doi.org/ $10.1063 \% 2 \mathrm{~F} 1.4908202$
. Transmission of urinary schistosomiasis in Sukumaland, Tanzania. 1. Snail infection rates and incidence of infection in school children. J Helminthol 1988;62(3):213-217. https://doi.org/10.10
and and incidence of infection in

15. Randjelovic A, Frønæs S, Munsami M, et al. A study of hurdles in mass treatment of schistosomiasis in KwaZuluNatal, South Africa. S Afr Fam Pract 2015;57(2):57-61. https://doi.org/10.1080\%2F20786190.2014.97812

16. South African Weather Service. Long Term Climatological Data for Paddock Weather Station. Durban: SAWS, 2012.

17. Kvalsvig J, Schutte C. The role of human water contact patterns in the transmission of schistosomiasis in an informal settlement near a major industrial area. Ann Trop Med Parasitol 1986;80(1):13-26. https://doi.org/10.1080\%2F00034983.1986.11811980

18. Hegertun I, Gundersen K, Kleppa E, et al. S. haematobium as a common cause of genital morbidity in girls: A cross-sectional study of children in South Africa. PLoS Negl Trop Dis 2013;7(3):e2104. https:// doi.org/10.1371\%2Fjournal.pntd.0002104

19. Agnew A, Fulford AJ, Mwanje MT, et al. Age-dependent reduction of schistosome fecundity in Schistosoma haematobium but not Schistosoma mansoni infections in humans. Am J Trop Med Hyg
Sy Schistosoma haematobium but not Schistosoma mansoni infection in

20. World Health Organization. WHO Urges Increased Access to Praziquantel as Schistosomiasis World Health Organization. WHO Urges Increase

21. Wilson S, Jones FM, van Dam GJ, et al. Human Schistosoma haematobium antifecundity immunity is dependent on transmission intensity and associated with immunoglobulin G1 to worm-derived antigens. J Infect Dis 2014;210(12):2009-2016. https://doi.org/10.1093\%2Finfdis\%2Fjiu374

22. Knopp S, Corstjens PL, Koukounari A, et al. Sensitivity and specificity of a urine circulating anodic antigen test for the diagnosis of Schistosoma haematobium in low endemic settings. PLoS Negl Trop Dis 2015;9(5):e0003752. https://doi.org/10.1371\%2Fjournal.pntd.0003752

23. Niangaly H, Djimde AA, Traore B, et al. [Seasonal variability of intestinal helminths and Schistosoma haematobium in a rural area of the Sahel in Mali.] Méd et santé Trop 2012;22(4):430-434. https://doi. org/10.1684/mst.2012.0123

24. Gbalégba N, Silué K, Ba O, et al. Prevalence and seasonal transmission of Schistosoma haematobium infection among school-aged children in Kaedi town, southern Mauritania. Parasites Vectors 2017;10(1):353. https://doi.org/10.1186/s13071-017-2284-4

25. Woolhouse ME, Taylor P, Matanhire D, Chandiwana SK. Acquired immunity and epidemiology of Schistosoma haematobium. Nature 1991;351(6329):757-759. https://doi.org/10.1038\%2F351757a0 